\section{(6) OPEN ACCESS}

\title{
[18F]Florbetapir positron emission tomography: identification of muscle amyloid in inclusion body myositis and differentiation from polymyositis
}

\author{
James B Lilleker, ${ }^{\oplus 1,2}$ Richard Hodgson, ${ }^{3,4}$ Mark Roberts, ${ }^{2}$ Karl Herholz, ${ }^{5}$ \\ James Howard, ${ }^{3}$ Rainer Hinz, ${ }_{1}^{5}$ Hector Chinoy ${ }^{\oplus, 6}$
}

\begin{abstract}
Handling editor Josef S Smolen

- Additional material is published online only. To view please visit the journal online (http://dx.doi.org/10.1136/ annrheumdis-2018-214644).
\end{abstract}

For numbered affiliations see end of article.

Correspondence to Dr James B Lilleker, Centre for Musculoskeletal Research, Stopford Building, Oxford Road, The University of Manchester, Manchester, UK.

james.lilleker@manchester.ac.uk

$\mathrm{RH}$ and $\mathrm{HC}$ contributed equally.

Received 25 October 2018 Revised 6 December 2018 Accepted 29 December 2018

Published Online First

13 February 2019

\section{ABSTRACT}

Objectives With the tools available currently, confirming the diagnosis of inclusion body myositis (IBM) can be difficult. Many patients are initially misdiagnosed with polymyositis (PM). In this observational study at a UK adult neuromuscular centre, we investigated whether amyloid positron emission tomography could differentiate between IBM and PM.

Methods Ten patients with IBM and six with PM underwent clinical review, [18F]florbetapir positron emission tomography and MRI of skeletal musculature. Differences in [18F]florbetapir standardised uptake value ratios in skeletal muscle regions of interest were evaluated. Relationships between [18F]florbetapir standardised uptake value ratios and measures of disease severity (clinical and by MRI of skeletal muscle) were assessed.

Results [18F]florbetapir standardised uptake value ratios were significantly higher in those with IBM compared with PM for all assessed regions (total-[18F] florbetapir standardised uptake value ratio 1.45 (1.28 to 2.05 ) vs 1.01 (0.80 to 1.22$), p=0.005)$. For total[18F]florbetapir standardised uptake value ratios $\geq 1.28$ sensitivity and specificity for IBM was $80 \%$ and $100 \%$, respectively.

Conclusions [18F]florbetapir amyloid positron emission tomography differentiates IBM from PM. Successful development could facilitate accurate diagnosis, inclusion in clinical trials and help avoid unnecessary exposure to potentially harmful treatments.

\section{INTRODUCTION}

Inclusion body myositis (IBM) is an acquired muscle disease with a slowly progressive course, culminating in severe disability. ${ }^{1}$ IBM is categorised as an inflammatory myopathy and shares histopathological features with polymyositis (PM), but immunosuppression does not modify progression. ${ }^{2}$ IBM is often diagnosed late and is commonly misdiagnosed initially as PM, due in part because differentiation on histopathological grounds can be difficult. In one study, five of nine patients with a diagnosis of 'PM' developed clinical features of IBM during follow-up, with such patients receiving unnecessary and potentially harmful immunosuppressive treatments. ${ }^{3}$

The presence of intramuscular beta-amyloid forms part of several IBM diagnostic criteria and is a key difference from PM. ${ }^{4}$ While this feature has a high diagnostic specificity, a relatively low

\section{Key messages}

What is already known about this subject?

- Positron emission tomography can detect tissue deposits of amyloid, potentially allowing non-invasive differentiation of inclusion body myositis (IBM) from polymyositis (PM).

What does this study add?

- Significantly increased intramuscular amyloid levels were found in IBM.

- Amyloid levels generally correlated poorly with disease severity, muscle inflammation and fatty infiltration levels.

How might this impact on clinical practice or future developments?

- Muscle amyloid imaging can differentiate between IBM and PM and could prove a useful future diagnostic modality.

sensitivity has been demonstrated, particularly in early IBM. ${ }^{5}$ Recent diagnostic criteria for IBM have shifted towards identification of the characteristic pattern of muscle weakness, with less strict histopathological requirements. ${ }^{4}$ While this has improved sensitivity, clinically detectable weakness implies that significant and irreversible muscle damage has occurred, reducing the likelihood that novel treatments will be effective.

We hypothesise that using amyloid positron emission tomography (amyloid-PET) to detect beta-amyloid within muscle can distinguish IBM from other inflammatory myopathies. Unlike muscle biopsy, imaging is non-invasive and large volumes of muscle can be studied, potentially improving sensitivity and facilitating earlier diagnosis. In this imaging study we compared the intramuscular amyloid burden, as determined using amyloid-PET, between IBM and PM. (E)-4-(2-(6-(2-(2-(2-18F-fluoroethoxy)ethoxy) ethoxy)pyridin-3-yl)vinyl)-N-methyl benzenamine, here referred to as [18F]florbetapir, was used as the amyloid imaging agent. ${ }^{67}$

\section{METHODS}

\section{Participants}

Between October 2015 and October 2016, written informed consent was provided by 10 cases with IBM and 6 with PM selected from the database of patients attending the adult neuromuscular service 
at Salford Royal NHS Foundation Trust, UK. For the PM cohort, we restricted recruitment to those aged $>45$ years (online supplementary appendix section 3). IBM cases met European Neuromuscular Centre 2011 diagnostic criteria ('clinicopathologically defined' $(n=8)$ or 'clinically defined' $(n=2)){ }^{8}$ Those with PM met Bohan and Peter diagnostic criteria (probable or definite) and had a minimum classification probability of $75 \%$ using the International Myositis Classification Criteria Project criteria. $^{9-11}$

\section{Study procedures}

\section{Clinical outcomes}

For those with IBM the Functional Rating Scale (IBM-FRS) was performed. ${ }^{12}$ In PM, the International Myositis Assessment \& Clinical Studies Group disease activity core set measures were completed. ${ }^{13}$ Both groups had muscle strength assessed using the manual muscle testing 260 (MMT26) score and completed the Health Assessment Questionnaire disability index (HAQ-DI). ${ }^{14}$

\section{PET}

A target dose of $370 \mathrm{MBq}(18 \mathrm{~F})$ florbetapir was administered by intravenous bolus. A CT scan from shoulders to ankles was performed using a Siemens Biograph TruePoint PET/CT camera for attenuation correction and definition of regions of interest (ROI). ${ }^{15}$ A PET emission scan of the same area commenced 45 min after radiotracer injection. Five minutes for each of the eight or nine bed positions was used, depending on subject height. PET images were reconstructed using 3D Ordered Subset Expectation Maximisation with three iterations and 21 subsets producing whole body images with almost isotropic voxels $(2.6728 \mathrm{~mm} \times 2.6728 \mathrm{~mm} \times 2.027 \mathrm{~mm})$ and a matrix size of $256 \times 256$ voxels per transaxial plane. A 3D Gaussian filter (full width at half maximum $3 \mathrm{~mm}$ ) was applied postreconstruction to regularise images.

\section{MRI}

On the same day, whole body MRI was performed on a Philips Achieva 1.5 T scanner. A T1-weighted (TR $500 \mathrm{~ms}$, TE $20 \mathrm{~ms}$, bandwidth $220 \mathrm{~Hz}$ ) sequence (to assess fatty infiltration of muscle) and a short tau inversion recovery (TR $5320 \mathrm{~ms}$, TE $50 \mathrm{~ms}$, TI $150 \mathrm{~ms}$, bandwidth $170 \mathrm{~Hz}$ ) sequence (to assess myoedema, a surrogate for muscle inflammation) were performed.

\section{Image processing}

\section{PET}

Seven muscle ROIs were defined for each subject, consisting of all muscle within a $10 \mathrm{~cm}$ vertical stack of consecutive images from the anatomical CT scan. The placement of this section was centred on a slice $1 / 3$ of the distance from the superior border of the patella to the anterior superior iliac spine for the thigh, $1 / 3$ of the distance from the inferior border of the patella to the summit of the medial malleolus for the calf, $1 / 2$ of the distance from the greater tuberosity of the humerus to the medial epicondyle for the left arm and 1/2 of the distance from the tip of the olecranon to the ulnar styloid process for the forearm. Each ROI was constructed using semiautomated threshold active contour segmentation tools within ITK-SNAP (online supplementary appendix section 1). ${ }^{16}$ Intensities of fat and muscle were specified (muscle: -10 to $+100 \mathrm{HU}$; fat: -150 to $-50 \mathrm{HU}$ ) and seed 'bubbles' placed within all visible musculature. Contour evolution could iterate until no further expansion of the ROI occurred.
For correction of non-specific radiotracer binding, a reference region was defined within the lumbar fat pad using the same centre landmark as the forearm ROI. Standardised [18F]florbetapir uptake values (SUVs) were calculated for each ROI by dividing the decay-corrected tissue mean concentration of radioactivity by the total injected radioactivity per body weight. Sum intensity means for all regions, upper limb regions and lower limb regions were calculated. SUV ratios (SUVRs) were calculated using the lumbar fat pad reference. This region was chosen as large volumes were available for selection and the location was easily matched between participants. Cerebral amyloid imaging studies have also shown increased statistical power when using lipid-rich reference regions. ${ }^{17}$ Given the lipophilic nature of florbetapir, it was assumed that tracer binding in the subcutaneous adipose was predominantly of the non-specific type.

\section{MRI}

Images were scored by a blinded musculoskeletal radiologist $(\mathrm{JH})$ using semiquantitative scoring tools based on those in the literature. ${ }^{18-20}$ Severity of fatty infiltration (0: normal, 5: end-stage appearance) and extent of inflammatory change (0: normal, 5: entire muscle) were scored (online supplementary appendix section 2). For comparison with the amyloid-PET, mean fatty infiltration and inflammation scores for corresponding muscle regions were calculated.

\section{Statistical analysis}

[18F]florbetapir SUVs and SUVRs for IBM were compared with PM using the Mann-Whitney Ranksum test in STATA for Windows V.13.0 (College Station, Texas, USA). For the IBM group, correlations between [18F]florbetapir SUVRs and clinical and MRI parameters of disease severity were examined using Spearman's ranked correlation. Two-sided students t-test or Fisher's exact test were used where appropriate. Receiver operating characteristic analysis was performed regarding the sensitivity and specificity of the total-[18F]florbetapir SUVRs for IBM. $\mathrm{P}<0.05$ was considered as significant. Disease duration refers to the interval between diagnosis and the date of participation in the study.

\section{Ethical and regulatory approvals}

The study was sponsored by the University of Manchester and authorised by the UK National Research Ethics Service (Greater Manchester West, 15/NW/0547) and the Administration of Radioactive Substances Advisory Committee (RPC number: 595/3586/33509).

\section{RESULTS}

Thirteen male and three female participants were studied (table 1). Three of the IBM group had previously received immunosuppressant medication, compared with all in the PM group. Visible differences were evident when comparing [18F]florbetapir PET/CT images between those with IBM and those with PM (figure 1). [18F]Florbetapir SUVRs were significantly higher in those with IBM for all ROIs (p value range 0.002-0.030) (table 1 and figure 2). For [18F]florbetapir SUVs (ie, without adjustment for non-specific radiotracer binding), only trends towards higher values in the IBM group were observed, except for the total-SUV region, where significantly higher values were also seen (table 1). For a total-[18F]florbetapir SUVR $\geq 1.28$ the diagnostic sensitivity for IBM was $80 \%$ and specificity $100 \%$ (area under curve 0.93). 
Table 1 Clinical characteristics of subjects and muscle [18F]florbetapir uptake values

\begin{tabular}{|c|c|c|c|c|}
\hline & & $\begin{array}{l}\text { IBM } \\
(n=10)\end{array}$ & $\begin{array}{l}P M \\
(n=6)\end{array}$ & $P$ value \\
\hline \multicolumn{2}{|l|}{ Mean age in years at diagnosis (SD) } & $64.3(8.4)$ & $58.2(10.7)$ & $0.222^{*}$ \\
\hline \multicolumn{2}{|l|}{ Mean age in years at scan (SD) } & $68.3(8.0)$ & $59.7(11.1)$ & $0.092^{*}$ \\
\hline \multicolumn{2}{|c|}{ Mean disease duration at scan in years (SD) } & $4.0(3.0)$ & $1.5(1.4)$ & $0.079^{*}$ \\
\hline \multicolumn{2}{|l|}{ Gender (Male | Female) } & $9 \mid 1$ & $4 \mid 2$ & $0.036 \dagger$ \\
\hline \multicolumn{2}{|c|}{ Mean manual muscle testing score $(0-260)(S D)$} & $236(22.9)$ & $256(2.3)$ & $0.052^{*}$ \\
\hline \multicolumn{2}{|c|}{ Mean Health Assessment Questionnaire disability index (SD) } & $1.3(0.7)$ & $0.8(0.8)$ & $0.192^{*}$ \\
\hline \multicolumn{2}{|c|}{ Mean IBM-Functional Rating Scale (0-40) (SD) } & $28.9(5.3)$ & - & - \\
\hline \multicolumn{2}{|c|}{ Mean physician global disease activity VAS (0-10) (SD) } & - & $1.8(1.5)$ & - \\
\hline \multicolumn{2}{|c|}{ Mean serum total creatine kinase level (IU/L) (SD) } & $579(408) \ddagger$ & $308(220)$ & - \\
\hline \multicolumn{2}{|c|}{ Current immunosuppressive treatments ( $\mathrm{n}$ ) } & Nil & $\begin{array}{l}\text { Prednisolone }(5 / 6) \\
\text { Methotrexate }(2 / 6) \\
\text { Azathioprine }(2 / 6) \\
\text { Cyclophosphamide }(1 / 6)\end{array}$ & - \\
\hline \multicolumn{2}{|c|}{ Previous immunosuppressive treatments ( $n$ ) } & $\begin{array}{l}\text { Prednisolone }(3 / 10) \\
\text { Azathioprine }(1 / 10) \\
\text { Mycophenolate }(1 / 10)\end{array}$ & $\begin{array}{l}\text { Cyclophosphamide (2/6) } \\
\text { Prednisolone }(1 / 6) \\
\text { Mycophenolate }(1 / 6) \\
\text { Azathioprine }(1 / 6) \\
\text { Ciclosporin }(1 / 6) \\
\text { IVIG }(1 / 6)\end{array}$ & - \\
\hline \multirow[t]{8}{*}{ Median [18F]florbetapir SUV (IQR) } & Left arm & $\begin{array}{l}0.47 \\
(0.41-0.55)\end{array}$ & $\begin{array}{l}0.40 \\
(0.36-0.48)\end{array}$ & $0.104 \S$ \\
\hline & Right forearm & $\begin{array}{l}0.39 \\
(0.35-0.42)\end{array}$ & $\begin{array}{l}0.32 \\
(0.27-0.40)\end{array}$ & $0.104 \S$ \\
\hline & Left forearm & $\begin{array}{l}0.45 \\
(0.32-0.55)\end{array}$ & $\begin{array}{l}0.33 \\
(0.30-0.36)\end{array}$ & $0.129 \S$ \\
\hline & Right thigh & $\begin{array}{l}0.44 \\
(0.43-0.52)\end{array}$ & $\begin{array}{l}0.41 \\
(0.37-0.45)\end{array}$ & $0.288 \S$ \\
\hline & Left thighף & $\begin{array}{l}0.48 \\
(0.43-0.51)\end{array}$ & $\begin{array}{l}0.41 \\
(0.36-0.45)\end{array}$ & $0.059 \S$ \\
\hline & Right calf & $\begin{array}{l}0.51 \\
(0.45-0.61)\end{array}$ & $\begin{array}{l}0.46 \\
(0.44-0.50)\end{array}$ & $0.233 \S$ \\
\hline & Left calf & $\begin{array}{l}0.51 \\
(0.40-0.58)\end{array}$ & $\begin{array}{l}0.43 \\
(0.39-0.45)\end{array}$ & $0.233 \S$ \\
\hline & Overall (total-SUV) & $\begin{array}{l}0.48 \\
(0.44-0.51)\end{array}$ & $\begin{array}{l}0.42 \\
(0.39-0.45)\end{array}$ & $0.039 \S$ \\
\hline \multirow[t]{8}{*}{ Median [18F]florbetapir SUVR (IQR) } & Left armๆ & $\begin{array}{l}1.61 \\
(1.43-1.81)\end{array}$ & $\begin{array}{l}0.96 \\
(0.82-1.08)\end{array}$ & $0.002 \S$ \\
\hline & Right forearm & $\begin{array}{l}1.26 \\
(1.05-1.60)\end{array}$ & $\begin{array}{l}0.79 \\
(0.67-0.91)\end{array}$ & $0.005 \S$ \\
\hline & Left forearm & $\begin{array}{l}1.26 \\
(1.12-1.52)\end{array}$ & $\begin{array}{l}0.83 \\
(0.58-0.96)\end{array}$ & $0.005 \S$ \\
\hline & Right thigh** & $\begin{array}{l}1.34 \\
(1.31-1.77)\end{array}$ & $\begin{array}{l}1.04 \\
(0.79-1.21)\end{array}$ & $0.013 \S$ \\
\hline & Left thigh** & $\begin{array}{l}1.40 \\
(1.40-1.87)\end{array}$ & $\begin{array}{l}0.99 \\
(0.79-1.18)\end{array}$ & $0.005 \S$ \\
\hline & Right calf & $\begin{array}{l}1.59 \\
(1.36-2.29)\end{array}$ & $\begin{array}{l}1.09 \\
(0.94-1.35)\end{array}$ & $0.013 \S$ \\
\hline & Left calf & $\begin{array}{l}1.56 \\
(1.29-2.40)\end{array}$ & $\begin{array}{l}1.00 \\
(0.75-1.31)\end{array}$ & $0.030 \S$ \\
\hline & Overall (total-SUVR) & $\begin{array}{l}1.45 \\
(1.28-2.05)\end{array}$ & $\begin{array}{l}1.01 \\
(0.80-1.22)\end{array}$ & $0.005 \S$ \\
\hline
\end{tabular}

Bold values indicate statistically significant differences.

${ }^{*} P$ values derive from two-sided students t-test.

†Fisher's exact test.

¥For the IBM group, this refers to the peak serum creatine kinase level (it was not rechecked at the time of the scan).

$\S$ The Mann-Whitney Ranksum test.

१The right arm was not used because radiotracer administration was via a venous cannula in the right antecubital fossa, except in two subjects (one with PM, one with IBM)

where the reverse was true due to difficulties with cannula placement.

${ }^{* *} \mathrm{n}=9$ for IBM group. Measurement in one subject could not be obtained due to very high levels of muscle atrophy and fatty replacement.

IBM, inclusion body myositis; IVIG, intravenous immunoglobulin; PM, polymyositis; SUV, standardised uptake value; SUVR, standardised uptake value ratio with reference region in lumbar fat pad; VAS, visual analogue scale. 


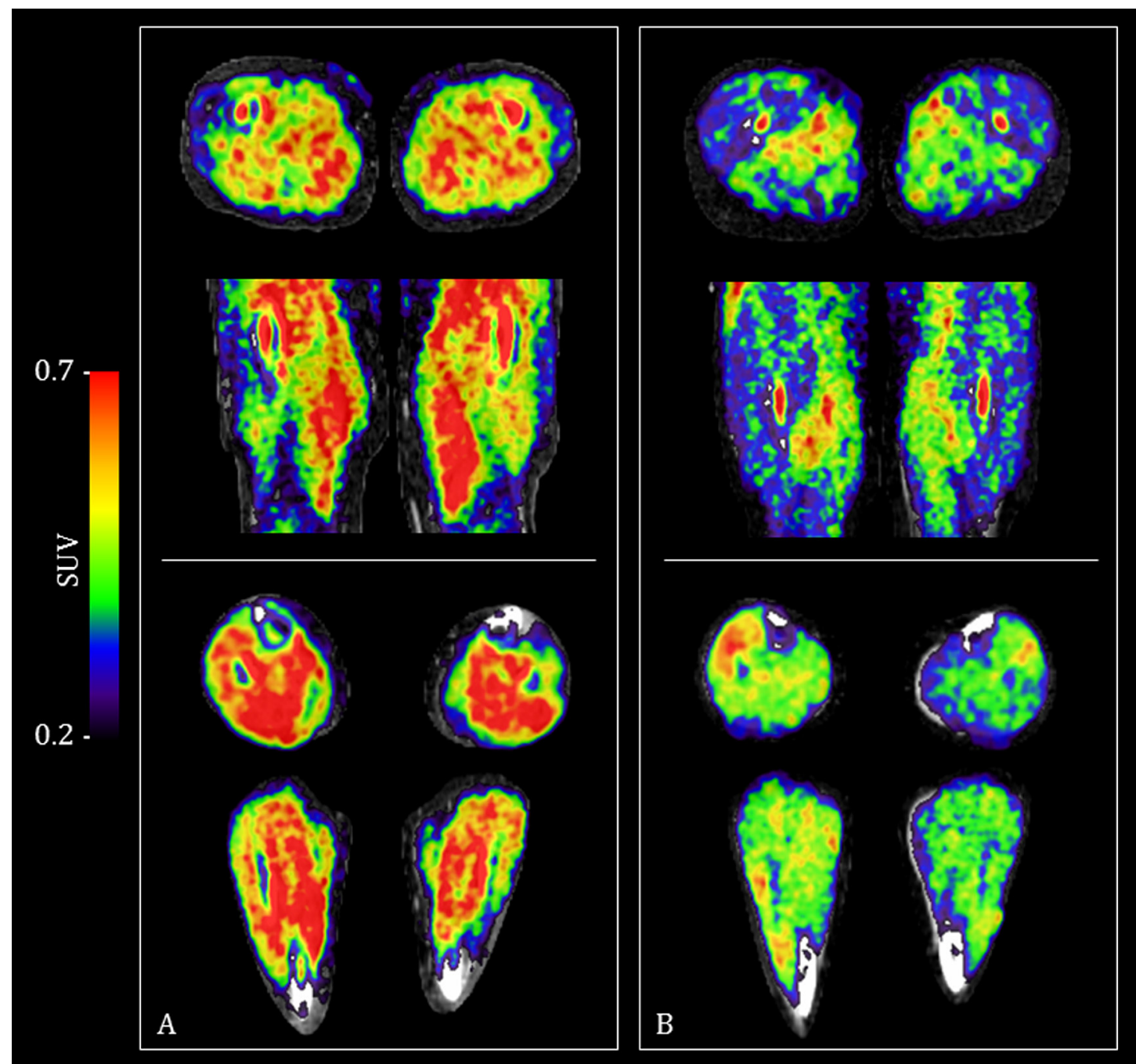

Figure 1 (18F)florbetapir PET/CT images showing differences in uptake between a participant with inclusion body myositis (panel A) and one with polymyositis (panel B). Increasing SUVs (red) indicate increased tracer uptake. [18F]Florbetapir PET images overlay spatially aligned CT images. Top of each panel depicts axial and coronal slices though the thigh. Bottom of each panel depicts axial and coronal slices though the calf. Each image is centred on the middle of the defined region of interest. PET, positron emission tomography; SUVs, standardised uptake values.

In those with IBM, only in the calves were strong negative correlations between $[18 \mathrm{~F}]$ florbetapir SUVRs and muscle inflammation levels (by MRI) found (right calf Rho -0.73 , $\mathrm{p}=0.02$; left calf Rho $-0.68, \mathrm{p}=0.03$ ). No significant correlation between $[18 \mathrm{~F}]$ florbetapir SUVRs and levels of fatty infiltration were identified. Furthermore, no significant relationships between the total-[18F]florbetapir SUVR and the age at scan, disease duration, MMT26, HAQ-DI or IBM-FRS were identified. This included subsets of the MMT26 and IBM-FRS restricted to upper limb and lower limb components compared with corresponding upper limb and lower limb [18F]florbetapir SUVRs (online supplementary appendix section 1 table 1 ). Amyloid deposits (by congo red staining) were only found in the diagnostic muscle biopsy of one IBM participant. No differences in the total-[18F]florbetapir SUVR were found according to the presence of degenerative biopsy features, including rimmed vacuoles (online supplementary appendix section 1 table 2).

\section{DISCUSSION}

In all assessed muscle groups, significantly increased [18F]florbetapir SUVRs were evident in IBM compared with PM. Sensitivity and specificity of the total-[18F]florbetapir SUVR for
IBM was high, highlighting the potential diagnostic usefulness of muscle amyloid-PET. Further development of this technique could facilitate accurate diagnosis of IBM in those with early and otherwise undifferentiated disease, avoiding the use of potentially harmful treatments and facilitating inclusion in clinical trials.

To our knowledge, only one other published study used PET to detect intramuscular amyloid in IBM. ${ }^{21}$ Maetzler et al used the Pittsburgh-B (PiB) compound; a carbon- 11 based radionucleotide with a half-life of approximately $20 \mathrm{~min}$ (compared with $110 \mathrm{~min}$ for fluorine-18), limiting its clinical use. Uniquely, we also performed same day muscle MRI and collected standardised clinical disease severity measures.

We used a semiautomated contour evolution method to select large sections of muscle for ROIs. ${ }^{16}$ It is likely that our method, rather than selecting small ellipsoid regions, produces more reliable results due to lower susceptibility to noise and bias from manual ROI placement. Borderline lower [18F]florbetapir SUVRs were found in the forearm when compared with other regions in both groups, potentially due to increased noise at the edge of the field of view. As we performed sequential exposures, comparison between different regions is susceptible to error, even after correction for radioactivity decay. 


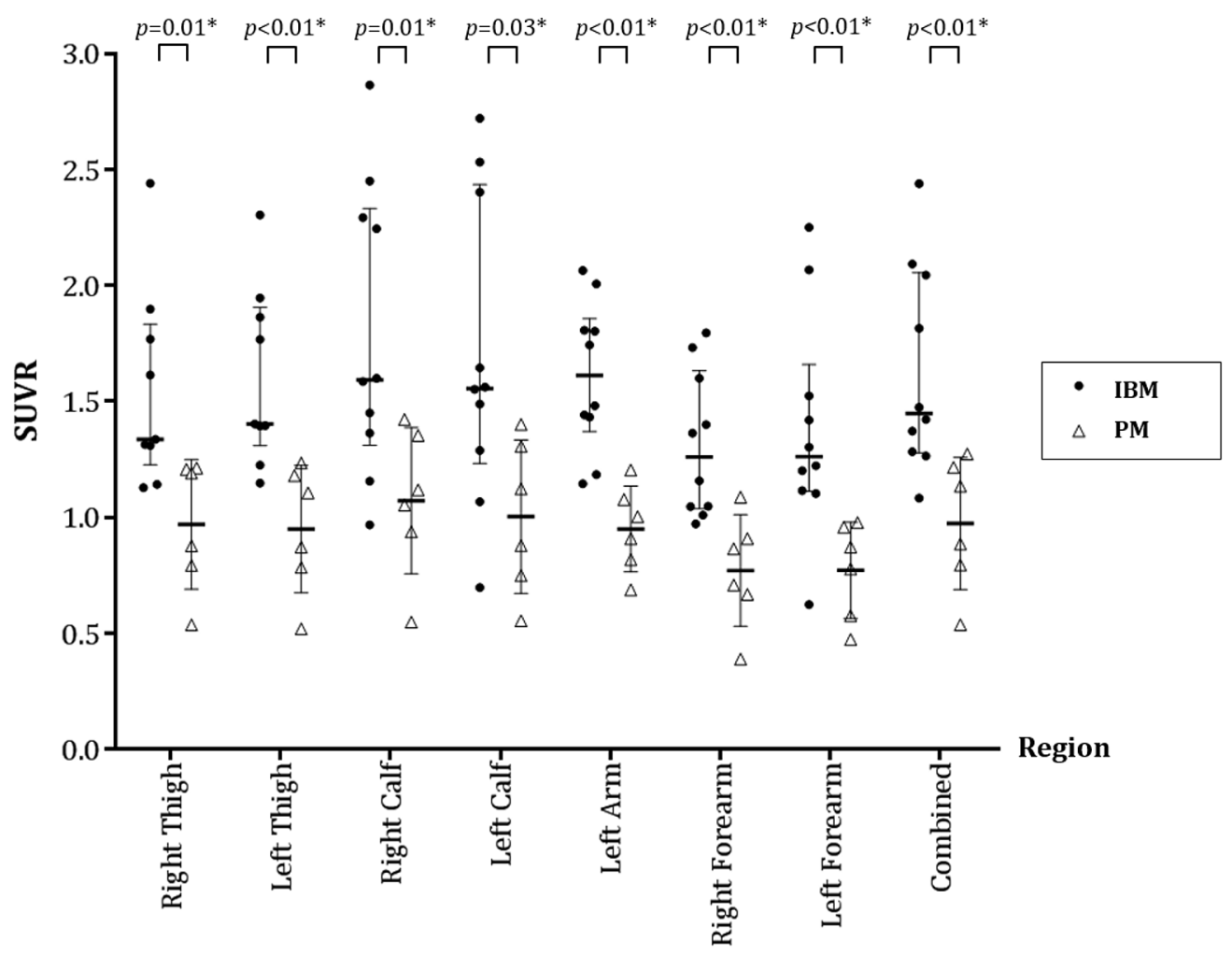

Figure 2 Comparison of SUVRs of [18F]florbetapir between participants with IBM (filled circles) and those with PM (open triangles) across seven different muscle regions and a combined region. Thick horizontal lines represent median SUVR and thin horizontal lines indicate the IQR. P values derived from Mann-Whitney Ranksum test. *Statistically significant difference $(p<0.05)$. IBM, inclusion body myositis; PM, polymyositis; SUVR, standardised uptake value ratio.

Our study is small and it is possible that factors other than diagnosis are confounding the results. A trend towards increased age at the time of scan is evident in the IBM group, but no significant correlations between age and the total-[18F]florbetapir SUVRs were evident $(\mathrm{Rho}=0.33, \mathrm{p}=0.22)$, indicating that age alone is unlikely to explain the differences in intramuscular amyloid content between the groups. The IBM group also had borderline lower MMT26 scores. However, total-[18F]florbetapir SUVRs did not correlate significantly with measures of disease severity in this group, including the MMT26. Gender ratios are also different between the groups, but we are not aware of a clear rationale as to why this would independently influence the $[18 \mathrm{~F}]$ florbetapir SUVR.

This study has demonstrated the usefulness of muscle amyloid imaging using $[18 \mathrm{~F}]$ florbetapir PET in differentiating IBM from PM. By potentially improving the ability to accurately diagnose IBM, further development and validation of this technique could help to avoid the use of unnecessary medication and enhance involvement in clinical trials.

\footnotetext{
Author affiliations

${ }^{1}$ Centre for Musculoskeletal Research, School of Biological Sciences, Faculty of Biology, Medicine and Health, Manchester Academic Health Science Centre, University of Manchester, Manchester, UK

${ }^{2}$ Manchester Centre for Clinical Neuroscience, Salford Royal NHS Foundation Trust, Salford, UK

${ }^{3}$ Radiology Department, Salford Royal NHS Foundation Trust, Salford, United Kingdom

${ }^{4}$ The National Institute for Health Research Manchester Musculoskeletal Biomedical Research Centre, Manchester University Hospitals NHS FoundationTrust, Manchester Academic Health Science Centre, University of Manchester, Manchester, United Kingdom

${ }^{5}$ Wolfson Molecular Imaging Centre, University of Manchester, Manchester, UK
}

${ }^{6}$ Rheumatology Department, Salford Royal NHS Foundation Trust, Salford, United Kingdom

Acknowledgements We thank all participants for kindly agreeing to take part in this study.

Collaborators Sarah Wood, Peter Julyan, David Ashworth, Gerrit van der Vegte, Mike Godfrey, Eleanor Duncan-Rouse, Barry Whitnall, Amy Watkins, Jonathan Harris, Mark Guy.

Contributors JBL and HC had the original idea for the project. JBL applied for funding, obtained ethical approval, designed the study, recruited and assessed subjects, analysed the data and drafted the manuscript. RH assisted with the design of the study, devised the PET scanning protocol and assisted with analysis of the data. RH assisted with the design of the study, devised the MRI scanning protocol and assisted with analysis of the data. MER and HC assisted with patient identification and recruitment. KH was the Administration of Radioactive Substances Advisory Committee (ARSAC) certificate holder. JH assisted with development of the MRI scoring system and scored the MRI scans. All authors contributed to, reviewed and approved the final manuscript.

Funding This work was supported by a grant from the NIHR Manchester Musculoskeletal Biomedical Research Unit (now Biomedical Research Centre), an award from the Centre for Imaging Sciences at the University of Manchester and a grant from the Medical Research Council (MR/N003322/1). This report includes independent research supported by the NIHR Biomedical Research Centre Funding Scheme.

Disclaimer The views expressed in this publication are those of the authors and not necessarily those of the NHS, the National Institute for Health Research or the Department of Health.

Competing interests None declared.

Patient consent for publication Not required.

Provenance and peer review Not commissioned; externally peer reviewed.

Open access This is an open access article distributed in accordance with the Creative Commons Attribution 4.0 Unported (CC BY 4.0) license, which permits others to copy, redistribute, remix, transform and build upon this work for any purpose, provided the original work is properly cited, a link to the licence is given, 
and indication of whether changes were made. See: https://creativecommons.org/ licenses/by/4.0/.

\section{REFERENCES}

1 Cox FM, Titulaer MJ, Sont JK, et al. A 12-year follow-up in sporadic inclusion body myositis: an end stage with major disabilities. Brain 2011;134:3167-75.

2 Rose MR, Jones K, Leong K, et al. Treatment for inclusion body myositis. Cochrane database Syst Rev 2015;51.

3 van der Meulen MF, Bronner IM, Hoogendijk JE, et al. Polymyositis: an overdiagnosed entity. Neurology 2003;61:316-21.

4 Lloyd TE, Mammen AL, Amato AA, et al. Evaluation and construction of diagnostic criteria for inclusion body myositis. Neurology 2014;83:426-33.

5 Brady S, Squier W, Hilton-Jones D. Clinical assessment determines the diagnosis of inclusion body myositis independently of pathological features. J Neurol Neurosurg Psychiatry 2013;84:1240-6.

6 Choi SR, Golding G, Zhuang Z, et al. Preclinical properties of 18F-AV-45: a PET agent for Abeta plaques in the brain. J Nucl Med 2009;50:1887-94.

7 Wong DF, Rosenberg PB, Zhou Y, et al. In vivo imaging of amyloid deposition in Alzheimer disease using the radioligand 18F-AV-45 (florbetapir [corrected] F 18). J Nucl Med 2010;51:913-20.

8 Rose MR, ENMC IBM Working Group. 188th ENMC International workshop: inclusion body myositis, 2-4 December 2011, Naarden, the Netherlands. Neuromuscul Disord 2013;23:1044-55.

9 Bohan A, Peter JB. Polymyositis and dermatomyositis (first of two parts). N Engl J Med 1975;292:344-7.

10 Bohan A, Peter JB. Polymyositis and dermatomyositis (second of two parts). N Eng/ J Med 1975:292:403-7.

11 Lundberg IE, Miller FW, Tjärnlund A, et al. Diagnosis and classification of idiopathic inflammatory myopathies. J Intern Med 2016:280:39-51.
12 Jackson CE, Barohn RJ, Gronseth G, et al. Inclusion body myositis functional rating scale: a reliable and valid measure of disease severity. Muscle Nerve 2008;37:473-6.

13 Rider LG, Werth VP, Huber AM, et al. Measures of adult and juvenile dermatomyositis, polymyositis, and inclusion body myositis: physician and patient/parent global activity, Manual Muscle Testing (MMT), Health Assessment Questionnaire (HAQ)/Childhood Health Assessment Questionnaire (C-HAQ). Arthritis Care Res 2011;63:S118-57.

14 Kendall FP, McCreary EK. Muscles, testing and function. Third Edit Williams \& Wilkins, 1983.

15 Jakoby BW, Bercier Y, Watson CC, et al. Performance characteristics of a new LSO PET/ CT scanner with extended axial Field-of-View and PSF reconstruction. IEEE Trans Nucl Sci 2009;56:633-9.

16 Yushkevich PA, Piven J, Hazlett HC, et al. User-guided 3D active contour segmentation of anatomical structures: significantly improved efficiency and reliability. Neuroimage 2006:31:1116-28.

17 Fleisher AS, Joshi AD, Sundell KL, et al. Use of white matter reference regions for detection of change in florbetapir positron emission tomography from completed phase 3 solanezumab trials. Alzheimer's \& Dementia 2017;13:1117-24.

18 Mercuri E, Talim B, Moghadaszadeh B, et al. Clinical and imaging findings in six cases of congenital muscular dystrophy with rigid spine syndrome linked to chromosome $1 p$ (RSMD1). Neuromuscul Disord 2002;12:631-8.

19 Yao L, Gai N. Fat-corrected T2 measurement as a marker of active muscle disease in inflammatory myopathy. AJR Am J Roentgenol 2012;198:W475-81.

20 Pinal-Fernandez I, Casal-Dominguez M, Carrino JA, et al. Thigh muscle MRI in immune-mediated necrotising myopathy: extensive oedema, early muscle damage and role of anti-SRP autoantibodies as a marker of severity. Ann Rheum Dis 2017;76:681-7.

21 Maetzler W, Reimold M, Schittenhelm J, et al. Increased [11C]PIB-PET levels in inclusion body myositis are indicative of amyloid beta deposition. J Neurol Neurosurg Psychiatry 2011;82:1060-2. 